\title{
Mean-Square Radius of Gyration of Poly(dimethylsiloxane) Chain with Side Groups ${ }^{\dagger}$
}

\author{
Haizhu $\mathrm{MA}^{\dagger \dagger}$ and Jianmin $\mathrm{XU}^{*}$ \\ Departmant of Mathematics-Physics, \\ Zhejiang Institute of Education, Hangzhou, \\ 310012, People's Republic of China \\ * Departmant of Physics, Hangzhou University, Hangzhou, \\ 310028, People's Republic of China
}

(Received August 4, 1993)

\begin{abstract}
Using the rotational isomeric state theory, the unperturbed mean-square radius of gyration $\left\langle S^{2}\right\rangle$ for the polymer chain considering the effect of a pair of side groups is derivated. If the spatial dimension (or the mass) of side groups and the mass difference of skeletal atoms are ignored, the expression is reduced to that of Flory and so forth. As an example, the numerical calculation of $\left\langle S^{2}\right\rangle$ is executed for unperturbed poly(dimethylsiloxane). The molecular weight dependence of the mean-square radius of gyration is $\left\langle S^{2}\right\rangle / M=7.82 \times 10^{-2} \AA^{2}(\mathrm{~g} \cdot \mathrm{mol})^{-1}$ which is in agreement with the experimental data. $\left\langle S^{2}\right\rangle / M=(7.7 \pm 0.3) \times 10^{-2} \AA^{2}(\mathrm{~g} \cdot \mathrm{mol})^{-1}$.

KEY WORDS Rotational Isomeric State / Mean-Square Radius of Gyration

/ Side Group / Poly(dimethylsiloxane) /
\end{abstract}

Several properties of polymers, such as the clasticity and flexiblity, are connected with the spatial dimension of the polymer chain, which is associated with the characteristic ratio of the mean-square end-to-end distance or the mean-square radius of gyration. The latter is particularly important to the branch polymer chain and the polymer with the large side groups. ${ }^{1,2}$ In addition, the mean-square radius of gyration can be readily evaluated from the light scattering or small-angle neutron scattering experiments. ${ }^{3-6}$ Therefore, the theoritical investigation of the mean-square radius of gyration for several polymers is very significant. The rotational isomeric state approximation (RIS) is the successful theory for treating configuration-dependent properties of chain molecules. $^{7-9}$ The mean-square radius of gyration, however, is customarily calculated by the Lagrange relation, ${ }^{7,9}$ in which only the structure of skeletal chain is accounted and the masses of skeletal atoms are regarded as identical. It is natural that the problem how to consider the effects of side groups and the mass difference of skeletal atom on it is attracted attention. Recently, the improved calculation of the mean-square radius of gyration for vinyl polymers considering a single side group has been reported. The results obtained for polypropylene, ${ }^{1}$ polystyrene, ${ }^{2}$ and polyethylene $\mathrm{e}^{10,11}$ are in good agreement with the experiment data. In this paper, the improved expression of mean-square radius of gyration for the chain polymer carrying a pair of side groups is further established by using the RIS theory.

The symmetrically substituted polysiloxanes consist of the monomeric units including

† The project supported by National Natural Science Foundation of China and Zhejiang Provincial Natural science Foundation of China.

${ }^{\dagger \dagger}$ To whom all correspondence should be addressed. 
the skeletal atoms ( $\mathrm{Si}$ and $\mathrm{O}$ ) and a pair of side groups, such as poly(dimethylsiloxane) (PDMS) with $-\left(\mathrm{CH}_{3}\right)_{2}$, poly(diethylsiloxane) with $-\left(\mathrm{C}_{2} \mathrm{H}_{5}\right)_{2}$ and poly(di- $n$-propylsiloxane) (PDNS) with $-\left(\mathrm{C}_{3} \mathrm{H}_{7}\right)_{2}$. The characteristic ratios reported by the experiment have a large difference between the PDMS and the PDNS. ${ }^{12-14}$ Therefore, the improved calculation may be necessary to investigating the effect of large side groups on the mean-square radius of gyration for several polymers. In this paper, the improved expression has been applied to evaluate the characteristic ratio of $\left\langle S^{2}\right\rangle$ for the PDMS as an example.

\section{EXPRESSION OF MEAN-SQUARE RADIUS OF GYRATION FOR THE POLYMER CHAIN WITH A PAIR OF SIDE GROUPS}

The mean-square radius of gyration for polymers is defined as

$$
\left\langle S^{2}\right\rangle=M^{-1} \sum_{j=1}^{4 x} m_{j}\left\langle s_{j}^{2}\right\rangle
$$

where $M$ is the molecular weight. In regard to the monosubstituted polymer chain with a pair of side groups as shown in Figure 1(a). $j$ is

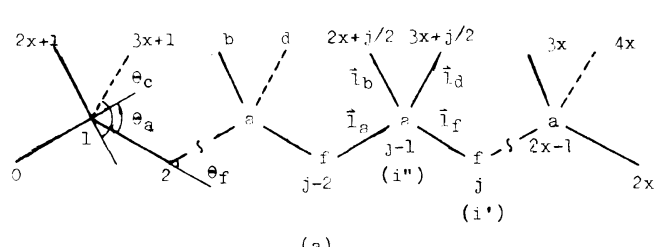

(a)

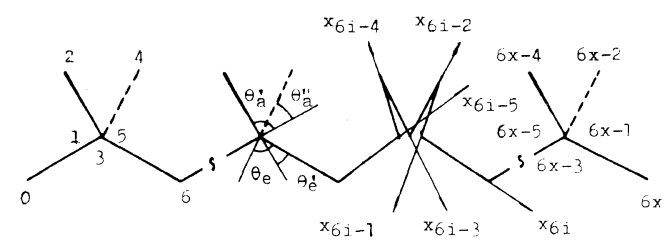

(b)

Figure 1. (a): A segmental model of the polymer chain with a pair of side groups in all-trans form. (b): Diagrammatic representation of the consecutive Cartesian reference frames affixed to the bonds.

taken from 0 to $2 x$ for the skeletal atoms and from $2 x+1$ to $4 x$ for side atoms, where $x$ is the degree of polymerization. $m_{\mathrm{j}}$ is the mass of atom(s) $j$. The masses of atoms a, b, d, and $\mathrm{f}$ in each repeat unit are $m_{\mathrm{a}}, m_{\mathrm{b}}, m_{\mathrm{d}}$, and $m_{\mathrm{f}}$, respectively. $s_{\mathrm{j}}$ is the distance of atom(s) $j$ from the center of mass of chain. Equation 1 can be also expressed as ${ }^{7}$

$$
\left\langle S^{2}\right\rangle=M^{-2}\left(\sum_{0 \leq i<j \leq 2 x}+\sum_{i=0}^{2 x} \sum_{j=2 x+1}^{4 x}+\sum_{i=2 x+1}^{3 x} \sum_{j=3 x+1}^{4 x}+\sum_{2 x+1 \leq i<j \leq 3 x}+\sum_{3 x+1 \leq i<j \leq 4 x}\right) m_{i} m_{j}\left\langle r_{i j}^{2}\right\rangle
$$

let

$$
\begin{gathered}
\left\langle S_{1}^{2}\right\rangle=\sum_{0 \leq i<j \leq 2 x} m_{i} m_{j}\left\langle r_{i j}^{2}\right\rangle \\
\left\langle S_{2}^{2}\right\rangle=\left(\sum_{i=0}^{2 x} \sum_{j=2 x+1}^{4 x}+\sum_{i=2 x+1}^{3 x} \sum_{j=3 x+1}^{4 x}+\sum_{2 x+1 \leq i<j \leq 3 x}+\sum_{3 x+1 \leq i<j \leq 4 x}\right) m_{i} m_{j}\left\langle r_{i j}^{2}\right\rangle
\end{gathered}
$$

where $r_{i j}$ is the distance from atom $i$ to $j$. It can be given by the bond vecter matrix $T_{j}$

$$
\bar{l}_{j}=\left[\begin{array}{lll}
l_{j} & 0 & 0
\end{array}\right]^{T}
$$

where $l_{j}$ is the bond length between atoms $j$ and $j+1$, and by the axis transformation matrix $T_{j}$ of the consecutive Cartesian reference frames $\left\{x_{j}, y_{j}, z_{j}\right\}$ affixed to the bonds

$$
\begin{aligned}
T_{j} & =\left[\begin{array}{ccc}
\cos \theta & \sin \theta & 0 \\
\sin \theta \cos \varphi & -\cos \theta \cos \varphi & \sin \varphi \\
\sin \theta \sin \varphi & -\cos \theta \sin \varphi & -\cos \varphi
\end{array}\right]_{j} \\
& =T\left(\theta_{j}, \varphi_{j}\right)
\end{aligned}
$$


where $\theta_{j}$ is the supplementary of bond angle for the atom $j, \varphi_{j}$ is the angle of internal rotation for bond $l_{j}$. According to the RIS theory, the rotational angle $\varphi_{j}$ can be taken several angles, such as three angles for three-state model, one for the trans conformation $(t)$ and two for the gauche conformations $\left(g^{ \pm}\right)$. Then, two statistical weight matrices $u_{\mathrm{a}}$ and $u_{\mathrm{f}}$ with orders $3 \times 3$ in each repeat unit are applicable to describe the isomeric state of chain.

The expression of $\left\langle S_{1}^{2}\right\rangle$ is only correlative to the sketetal chain. Following the RIS theory, it can be formulated as

$\left\langle S_{1}^{2}\right\rangle$

$$
=2 Z^{-1} \sum_{0 \leq j^{\prime}<j \leq 2 x} m_{j^{\prime}} m_{j} J^{*} u_{1}^{\left(j^{\prime}\right)} P g_{j^{\prime}+1}^{\left(j-j^{\prime}\right)} Q u_{j+1}^{(2 x-j)} J
$$

where

$$
\begin{aligned}
& J^{*}=\left[\begin{array}{lll}
1 & 0 & 0
\end{array}\right], \quad J=\left[\begin{array}{lll}
1 & 1 & 1
\end{array}\right]^{T} \\
& P=\left[\begin{array}{llll}
E_{3} & 0 & \cdots & 0
\end{array}\right], \quad Q=\left[\begin{array}{llll}
0 & \cdots & 0 & E_{3}
\end{array}\right]^{T}
\end{aligned}
$$

with $E_{3}$ being the identity matrix of orders 3 . The partition function $Z$ and the generator matrix $g_{j}$ are

$$
\begin{aligned}
& Z=J^{*} u_{1}^{(2 x)} J=J^{*}\left(u_{\mathrm{f}} u_{\mathrm{a}}\right)^{x-1} J \\
g_{j}= & {\left[\begin{array}{ccc}
u_{j} & \left(u_{j} \otimes I_{j}^{T}\right)\|T\|_{j} & \left(l_{j}^{2} / 2\right) u_{j} \\
0 & \left(u_{j} \otimes E_{3}\right)\|T\|_{j} & u_{j} \otimes \bar{l}_{j} \\
0 & 0 & u_{j}
\end{array}\right] } \\
= & g\left(u_{j}, \bar{l}_{j}, T_{j}\right)
\end{aligned}
$$

where

$$
\|T\|_{j}=\left[\begin{array}{ccc}
T\left(\varphi_{\mathrm{t}}\right) & 0 & 0 \\
0 & T\left(\varphi_{g^{+}}\right) & 0 \\
0 & 0 & T\left(\varphi_{g^{-}}\right)
\end{array}\right]_{j}
$$

$P, Q, g_{j}$, and $\|T\|_{j}$ are the matrices of orders $3 \times 15,15 \times 3,15 \times 15$, and $9 \times 9$, respectively. $j$ is taken $1,3 \cdots 2 x-1$ for the skeletal atom a and $2,4 \cdots 2 x$ for $f$. Then, eq 7 can be simplified as

$$
\begin{aligned}
\left\langle S_{1}^{2}\right\rangle & =2 Z^{-1} F^{*} \prod_{j=1}^{2 x} G_{j} F \\
& =2 Z^{-1} F^{*}\left(G_{\mathrm{a}} G_{\mathrm{f}}\right)_{1}\left(G_{\mathrm{a}} G_{\mathrm{f}}\right)^{x-2}\left(G_{\mathrm{a}} G_{\mathrm{f}}\right)_{x} F
\end{aligned}
$$

where

$$
\begin{aligned}
& F^{*}=\left[\begin{array}{llll}
1 & 0 & \cdots & 0
\end{array}\right], \quad F=\left[\begin{array}{llllll}
0 & \cdots & 0 & 1 & 1 & 1
\end{array}\right]^{T} \\
& G_{\mathrm{a}}=\left[\begin{array}{ccc}
u_{\mathrm{a}} & m_{\mathrm{f}} P g_{\mathrm{a}} & m_{\mathrm{a}} m_{\mathrm{f}}\left(l_{\mathrm{a}}^{2} / 2\right) u_{\mathrm{a}} \\
0 & g_{\mathrm{a}} & m_{\mathrm{a}} g_{\mathrm{a}} Q \\
0 & 0 & u_{\mathrm{a}}
\end{array}\right] \\
& G_{\mathrm{f}}=\left[\begin{array}{ccc}
u_{\mathrm{f}} & m_{\mathrm{a}} P g_{\mathrm{f}} & m_{\mathrm{a}} m_{\mathrm{f}}\left(l_{\mathrm{f}}^{2} / 2\right) u_{\mathrm{f}} \\
0 & g_{\mathrm{f}} & m_{\mathrm{f}} g_{\mathrm{f}} Q \\
0 & 0 & u_{\mathrm{f}}
\end{array}\right]
\end{aligned}
$$

$F^{*}, F$, and $G_{\mathrm{a}}$ or $G_{\mathrm{f}}$ are the matrices with orders $1 \times 21,21 \times 1$, and $21 \times 21$, respectively. $u_{\mathrm{a}}$ in $G_{\mathrm{a}}$ of the chain terminal 1 or $u_{\mathrm{f}}$ in $G_{\mathrm{f}}$ of the terminal $x$ is replaced with the identity matrix $E_{3}$.

The expression of $\left\langle S_{2}^{2}\right\rangle$ is related to the structure of side groups $b$ and $d$ in every repeat units. Therefore, the marks from 0 to $4 x$ in Figure 1(a) are renumbered serially from 0 to $6 x$, moreover, the Cartesian reference frames $\left\{x_{j}, y_{j}, z_{j}\right\}$ affixed to the skeletal bonds and side bonds are founded as shown in Figure 1(b), where $j$ is taken $6 i-5,6 i-4,6 i-3,6 i-2,6 i-1$, and $6 i$ in the $i$-th repeat unit, or expressed as $\mathrm{a}, \mathrm{b}, \mathrm{c}, \mathrm{d}, \mathrm{e}$, and $\mathrm{f}$ in every repeat units, and $i$ is taken from 1 to $x$. Let the axis $x_{6 i-5}, x_{6 i-4}$, $x_{6 i-2}$, and $x_{6 i}$ affixed to the bonds $l_{\mathrm{a}}, l_{\mathrm{b}}, l_{\mathrm{d}}$, and $l_{\mathrm{f}}$ be in the direction of the bond vectors $\boldsymbol{l}_{\mathrm{a}}, \boldsymbol{l}_{b}$, $\boldsymbol{l}_{\mathrm{d}}$, and $\boldsymbol{l}_{\mathrm{f}}$, respectively. The axis $x_{6 i-3}$ and $x_{6 i-1}$ affixed to the bonds $l_{\mathrm{b}}$ and $l_{\mathrm{d}}$ in the oppesite direction of bond vectors $\boldsymbol{l}_{\mathrm{b}}$ and $\boldsymbol{l}_{\mathrm{d}}$, respectively. The axis $y_{j}$ and $z_{j}$ of consecutive coordinate systems are defined in similar way to the RIS theory. Then, $\theta_{\mathrm{a}}{ }^{\prime}, \theta_{\mathrm{c}}, \theta_{\mathrm{e}}$, or $\theta_{\mathrm{f}}$ are the angles between axis $x_{6 i-5}$ and $x_{6 i-4}, x_{6 i-3}$, and $x_{6 i-2}$, $x_{6 i-1}$, and $x_{6 i}$ or $x_{6 i}$ and $x_{6 i+1}$, respectively. $\varphi_{j}$ in eq 6 may be separated into two parts $\varphi_{j}$ and $\varphi_{j}^{\prime}$. The former describes the states of internal rotation of bond $l_{j}$. The latter depended on the geometric structure of 
polymer chain denotes the dihedral angle between plan $x_{j} y_{j}$ and plan $x_{j+1} y_{j+1}$ when the angles of internal rotation are in trans form. Therefore, after considering the geometric structure of side groups, the consecutive axis transformation matrix $T_{j}$ in each repeat unit is also given by eq 6

$$
\begin{aligned}
& T_{6 i-5}=T_{\mathrm{a}}=T\left(\theta_{\mathrm{a}}{ }^{\prime}, \varphi_{\mathrm{a}}+\varphi_{\mathrm{a}}{ }^{\prime}\right) \\
& T_{6 i-4}=T_{\mathrm{b}}=T\left(180^{\circ}, 0^{\circ}\right) \\
& T_{6 i-3}=T_{\mathrm{c}}=T\left(\theta_{\mathrm{c}}, \varphi_{\mathrm{c}}{ }^{\prime}\right) \\
& T_{6 i-2}=T_{\mathrm{d}}=T\left(180^{\circ}, 0^{\circ}\right) \\
& T_{6 i-1}=T_{\mathrm{e}}=T\left(\theta_{\mathrm{e}}, \varphi_{\mathrm{e}}{ }^{\prime}\right) \\
& T_{6 i}=T_{\mathrm{f}}=T\left(\theta_{\mathrm{f}}, \varphi_{\mathrm{f}}+\varphi_{\mathrm{f}}{ }^{\prime}\right)
\end{aligned}
$$

where $\varphi_{\mathrm{a}}{ }^{\prime}, \varphi_{\mathrm{c}}{ }^{\prime}, \varphi_{\mathrm{e}}{ }^{\prime}$, and $\varphi_{\mathrm{f}}{ }^{\prime}$ can be calculated by

$$
\begin{aligned}
\cos \varphi_{\mathrm{a}}{ }^{\prime}= & -\left(\cos \theta_{\mathrm{c}}+\cos \theta_{\mathrm{a}}{ }^{\prime} \cos \theta_{\mathrm{a}}{ }^{\prime \prime}\right) \\
& / \sin \theta_{\mathrm{a}}{ }^{\prime} \sin \theta_{\mathrm{a}}{ }^{\prime \prime}
\end{aligned}
$$

$$
\begin{aligned}
\cos \varphi_{\mathrm{c}}{ }^{\prime}= & \left(\cos \theta_{\mathrm{a}}{ }^{\prime \prime}+\cos \theta_{\mathrm{a}}{ }^{\prime} \cos \theta_{\mathrm{c}}\right) \\
& / \sin \theta_{\mathrm{a}}{ }^{\prime} \sin \theta_{\mathrm{c}} \\
\cos \varphi_{\mathrm{e}}{ }^{\prime}= & \left(\cos \theta_{\mathrm{e}}{ }^{\prime}+\cos \theta_{\mathrm{c}} \cos \theta_{\mathrm{e}}\right) \\
& / \sin \theta_{\mathrm{c}} \sin \theta_{\mathrm{e}} \\
\cos \varphi_{\mathrm{f}}{ }^{\prime}= & -\left(\cos \theta_{\mathrm{f}}{ }^{\prime}+\cos \theta_{\mathrm{e}} \cos \theta_{\mathrm{f}}\right) \\
& / \sin \theta_{\mathrm{e}} \sin \theta_{\mathrm{f}}
\end{aligned}
$$

where $\theta_{\mathrm{a}}{ }^{\prime \prime}$ or $\theta_{\mathrm{e}}{ }^{\prime}$ are the angles between axis $x_{6 i-5}$ and $x_{6 i-2}$ or $x_{6 i-3}$ and $x_{6 i}$, respectively. $\theta_{\mathrm{f}}{ }^{\prime}$ is the angle between axis $x_{6 i-2}$ and $x_{6 i+1}$. The statistical weight matrix $u_{j}$ is expressed as

$$
\begin{gathered}
u_{6 i-5}=u_{\mathrm{a}} \quad(i=2,3, \cdots, x) \\
u_{j}=E_{3} \quad \begin{array}{l}
(j=6 i-4,6 i-3,6 i-2 ; 6 i-1 ; \\
i=1,2, \cdots, x \text { and } j=1,6 x)
\end{array} \\
u_{6 i}=u_{\mathrm{f}} \quad(i=1,2, \cdots, x-1)
\end{gathered}
$$

$\left\langle S_{2}^{2}\right\rangle$ can be derivated by comparing (a) with (b) in Figure 1 as

$$
\begin{aligned}
\left\langle S_{2}^{2}\right\rangle= & \left(\sum_{i=0}^{2 x} \sum_{j=2 x+1}^{4 x}+\sum_{i=2 x+1}^{3 x} \sum_{j=3 x+1}^{4 x}+\sum_{2 x+1 \leq j \leq 3 x}+\sum_{3 x+1 \leq i<j \leq 4 x}\right) m_{i} m_{j}\left\langle r_{i j}^{2}\right\rangle \\
= & \left(m_{\mathrm{f}} m_{\mathrm{b}} \sum_{i^{\prime}=0}^{x} \sum_{j=2 x+1}^{3 x}+m_{\mathrm{f}} m_{\mathrm{d}} \sum_{i^{\prime}=0}^{x} \sum_{j=3 x+1}^{4 x}\right)\left\langle r_{i j}^{2}\right\rangle \\
& +\left(m_{\mathrm{a}} m_{\mathrm{b}} \sum_{i^{\prime \prime}=1}^{x} \sum_{j=2 x+1}^{3 x}+m_{\mathrm{a}} m_{\mathrm{d}} \sum_{i^{\prime \prime}=1}^{x} \sum_{j=3 x+1}^{4 x}\right)\left\langle r_{\mathrm{ij}}^{2}\right\rangle \\
& +\left(m_{\mathrm{b}} m_{\mathrm{d}} \sum_{i=2 x+1}^{3 x} \sum_{j=3 x+1}^{4 x}+m_{\mathrm{b}}^{2} \sum_{2 x+1 \leq i<j \leq 3 x}+m_{\mathrm{d}}^{2} \sum_{3 x+1 \leq i<j \leq 4 x}\right)\left\langle r_{i j}^{2}\right\rangle \\
= & m_{\mathrm{f}} m_{\mathrm{b}} \sum_{h=0}^{x} \sum_{k=1}^{x}\left\langle r_{6 h, 6 k-4}^{2}\right\rangle+m_{\mathrm{f}} m_{\mathrm{d}} \sum_{h=0}^{x} \sum_{k=1}^{x}\left\langle r_{6 h, 6 k-2}^{2}\right\rangle \\
& +m_{\mathrm{a}} m_{\mathrm{b}} \sum_{h=1}^{x} \sum_{k=1}^{x}\left\langle r_{6 h-5,6 k-4}^{2}\right\rangle+m_{\mathrm{a}} m_{\mathrm{d}} \sum_{h=1}^{x} \sum_{k=1}^{x}\left\langle r_{6 h-5,6 k-2}^{2}\right\rangle \\
& +m_{\mathrm{b}} m_{\mathrm{d}} \sum_{h=1}^{x} \sum_{k=1}^{x}\left\langle r_{6 h-4,6 k-2}^{2}\right\rangle+m_{\mathrm{b}}^{2} \sum_{1 \leq h<k \leq x}\left\langle r_{6 h-4,6 k-4}^{2}\right\rangle \\
& +m_{\mathrm{d}}^{2} \sum_{1 \leq h<k \leq x}\left\langle r_{6 h-2,6 k-2}^{2}\right\rangle
\end{aligned}
$$

where

$$
\left.\begin{array}{ll}
i^{\prime}=j / 2 & (j=0,2,4 \cdots 2 x) \\
i^{\prime \prime}=(j+1) / 2 & (j=1,3,5 \cdots 2 x-1)
\end{array}\right\}
$$

Let the generator matrix $g_{j}$ from eq 11 in the $i$-th unit 


$$
\begin{aligned}
& g_{6 i-5}=g_{\mathrm{a}}=g\left(u_{\mathrm{a}}, T_{\mathrm{a}}, T_{\mathrm{a}}\right) \\
& g_{6 i-4}=g_{\mathrm{b}}=g\left(E_{3}, T_{\mathrm{b}}, T_{\mathrm{b}}\right) \\
& g_{6 i-3}=g_{\mathrm{c}}=g\left(E_{3}, T_{\mathrm{b}}, T_{\mathrm{c}}\right) \\
& g_{6 i-2}=g_{\mathrm{d}}=g\left(E_{3}, T_{\mathrm{d}}, T_{\mathrm{d}}\right) \\
& g_{6 i-1}=g_{\mathrm{e}}=g\left(E_{3}, T_{\mathrm{d}}, T_{\mathrm{e}}\right) \\
& g_{6 i}=g_{\mathrm{f}}=g\left(u_{\mathrm{f}}, \bar{l}_{\mathrm{f}}, T_{\mathrm{f}}\right)
\end{aligned}
$$

then, eq 25 is given by using the matrix algebra method

$$
\begin{aligned}
\left\langle S_{2}^{2}\right\rangle= & 2 Z^{-1}\left[m_{\mathrm{f}} m_{\mathrm{b}} F^{*} S(1)_{1} S(1)^{x-2} S(1)_{x} F\right. \\
& +2 m_{\mathrm{f}} m_{\mathrm{d}} F^{*} S(2)_{1} S(2)^{x-2} S(2)_{x} F \\
& +2 m_{\mathrm{a}} m_{\mathrm{b}} F^{*} S(3)_{1} S(3)^{x-2} S(3)_{x} F \\
& +2 m_{\mathrm{a}} m_{\mathrm{d}} F^{*} S(4)_{1} S(4)^{x-2} S(4)_{x} F \\
& +2 m_{\mathrm{b}} m_{\mathrm{d}} F^{*} S(5)_{1} S(5)^{x-2} S(5)_{x} F \\
& +m_{\mathrm{b}}^{2} F^{*} S(6)_{1} S(6)^{x-2} S(6)_{x} F \\
& \left.+m_{\mathrm{d}}^{2} F^{*} S(7)_{1} S(7)^{x-2} S(7)_{x} F\right]
\end{aligned}
$$

where

$$
\begin{aligned}
& S(1)=\left[\begin{array}{ccc}
u_{\mathrm{a}} u_{\mathrm{f}} & P g_{\mathrm{a}} g_{\mathrm{b}} g_{\mathrm{c}} g_{\mathrm{d}} g_{\mathrm{e}} g_{\mathrm{f}} & P g_{\mathrm{a}} g_{\mathrm{b}} Q u_{\mathrm{f}} \\
0 & g_{\mathrm{a}} g_{\mathrm{b}} g_{\mathrm{c}} g_{\mathrm{d}} g_{\mathrm{e}} g_{\mathrm{f}} & g_{\mathrm{a}} g_{\mathrm{b}} Q u_{\mathrm{f}} \\
0 & 0 & u_{\mathrm{a}} u_{\mathrm{f}}
\end{array}\right] \\
& S(2)=\left[\begin{array}{ccc}
u_{\mathrm{a}} u_{\mathrm{f}} & P g_{\mathrm{a}} g_{\mathrm{b}} g_{\mathrm{c}} g_{\mathrm{d}} g_{\mathrm{e}} g_{\mathrm{f}} P g_{\mathrm{a}} g_{\mathrm{b}} g_{\mathrm{c}} g_{\mathrm{d}} Q u_{\mathrm{f}} \\
0 & g_{\mathrm{a}} g_{\mathrm{b}} g_{\mathrm{c}} g_{\mathrm{d}} g_{\mathrm{e}} g_{\mathrm{f}} & g_{\mathrm{a}} g_{\mathrm{b}} g_{\mathrm{c}} g_{\mathrm{d}} Q u_{\mathrm{f}} \\
0 & 0 & u_{\mathrm{a}} u_{\mathrm{f}}
\end{array}\right] \\
& S(3)=\left[\begin{array}{ccc}
u_{\mathrm{a}} u_{\mathrm{f}} & u_{\mathrm{a}} P g_{\mathrm{b}} g_{\mathrm{c}} g_{\mathrm{d}} g_{\mathrm{e}} g_{\mathrm{f}} & \left(l_{\mathrm{b}}^{2} / 4\right) u_{\mathrm{a}} u_{\mathrm{f}} \\
0 & g_{\mathrm{a}} g_{\mathrm{b}} g_{\mathrm{c}} g_{\mathrm{d}} g_{\mathrm{e}} g_{\mathrm{f}} & g_{\mathrm{a}} g_{\mathrm{b}} Q u_{\mathrm{f}} \\
0 & 0 & u_{\mathrm{a}} u_{\mathrm{f}}
\end{array}\right] \\
& S(4)=\left[\begin{array}{ccc}
u_{\mathrm{a}} u_{\mathrm{f}} & u_{\mathrm{a}} P g_{\mathrm{b}} g_{\mathrm{c}} g_{\mathrm{d}} g_{\mathrm{e}} g_{\mathrm{f}} & \left(l_{\mathrm{d}}^{2} / 4\right) u_{\mathrm{a}} u_{\mathrm{f}} \\
0 & g_{\mathrm{a}} g_{\mathrm{b}} g_{\mathrm{c}} g_{\mathrm{d}} g_{\mathrm{e}} g_{\mathrm{f}} & g_{\mathrm{a}} g_{\mathrm{b}} g_{\mathrm{c}} g_{\mathrm{d}} Q u_{\mathrm{f}} \\
0 & 0 & u_{\mathrm{a}} u_{\mathrm{f}}
\end{array}\right] \\
& S(5)=\left[\begin{array}{ccc}
u_{\mathrm{a}} u_{\mathrm{f}} & u_{\mathrm{a}} P g_{\mathrm{c}} g_{\mathrm{d}} g_{\mathrm{e}} g_{\mathrm{f}} & \left(r_{\mathrm{bd}}^{2} / 4\right) u_{\mathrm{a}} u_{\mathrm{f}} \\
0 & g_{\mathrm{a}} g_{\mathrm{b}} g_{\mathrm{c}} g_{\mathrm{d}} g_{\mathrm{e}} g_{\mathrm{f}} & g_{\mathrm{a}} g_{\mathrm{b}} g_{\mathrm{c}} g_{\mathrm{d}} Q u_{\mathrm{f}} \\
0 & 0 & u_{\mathrm{a}} u_{\mathrm{f}}
\end{array}\right]
\end{aligned}
$$

$$
\begin{aligned}
& S(6)=\left[\begin{array}{ccc}
u_{\mathrm{a}} u_{\mathrm{f}} & u_{\mathrm{a}} P g_{\mathrm{c}} g_{\mathrm{d}} g_{\mathrm{e}} g_{\mathrm{f}} & 0 \\
0 & g_{\mathrm{a}} g_{\mathrm{b}} g_{\mathrm{c}} g_{\mathrm{d}} g_{\mathrm{e}} g_{\mathrm{f}} & g_{\mathrm{a}} g_{\mathrm{b}} Q u_{\mathrm{f}} \\
0 & 0 & u_{\mathrm{a}} u_{\mathrm{f}}
\end{array}\right] \\
& S(7)=\left[\begin{array}{ccc}
u_{\mathrm{a}} u_{\mathrm{f}} & u_{\mathrm{a}} P g_{\mathrm{e}} g_{\mathrm{f}} & 0 \\
0 & g_{\mathrm{a}} g_{\mathrm{b}} g_{\mathrm{c}} g_{\mathrm{d}} g_{\mathrm{e}} g_{\mathrm{f}} & g_{\mathrm{a}} g_{\mathrm{b}} g_{\mathrm{c}} g_{\mathrm{d}} Q u_{\mathrm{f}} \\
0 & 0 & u_{\mathrm{a}} u_{\mathrm{f}}
\end{array}\right]
\end{aligned}
$$

Then, the unperturbed mean-square radius of gyration of the polymer chain considering the pair side groups can be formulated by substitution of eq 13 and 28 into eq 2 as

$$
\begin{aligned}
& \left\langle S^{2}\right\rangle=2 Z^{-1} M^{-2} F^{*}\left(G_{\mathrm{a}} G_{\mathrm{f}}\right)_{1}\left(G_{\mathrm{a}} G_{\mathrm{f}}\right)^{x-2}\left(G_{\mathrm{a}} G_{\mathrm{f}}\right)_{x} F \\
& +2 Z^{-1} M^{-2} \sum_{q=1}^{7} m(q) F^{*} S(q)_{1} S(q)^{x-2} S(q)_{x} F(36)
\end{aligned}
$$

where $m(1)-m(7)$ have replaced $2 m_{\mathrm{f}} m_{\mathrm{b}}, 2 m_{\mathrm{f}} m_{\mathrm{d}}$, $2 m_{\mathrm{a}} m_{\mathrm{b}}, 2 m_{\mathrm{a}} m_{\mathrm{d}}, 2 m_{\mathrm{b}} m_{\mathrm{d}}, m_{\mathrm{b}}^{2}$, and $m_{\mathrm{d}}^{2}$, respectively. $u_{\mathrm{a}}$ in the terminal matrix $S(q)_{1}$ and $u_{\mathrm{f}}$ in matrix $S(q)_{x}$ are replaced with $E_{3}$. Obviously, the seconed term in eq. 36 is related to the dimension and masses of side groups. If the size of the pair side groups is ignored, i.e., the length of bonds $l_{\mathrm{b}}$ and $l_{\mathrm{d}}$ are assumed to be zero, $g_{j}(j$ is taken $b, c, d$, and $e$ ) of eq 11 becomes the diagonal matrix. The second term in eq 36 becomes zero because $P \prod_{j} g_{j} Q$ gives the zero matrix of orders 3 . In another way, the masses of side groups $b$ and $d$ are assumed to be zero, the seconed term also becomes zero. Further, if the skeletal atoms are regarded as identical, the first term retained in eq 36 is reduced to that by Flory. ${ }^{8,10}$ Similarly, if the length of bonds $l_{\mathrm{b}}$ (or $l_{\mathrm{d}}$ ) or the mass of atom $\mathrm{b}$ (or d) is assumed to be zero. Equation 36 is reduced that considering a single side groups reported by ref 1 .

\section{NUMERICAL RESULTS FOR POLY- (DIMETHYLSILOXANE)}

The two statistical weight matrices only considering the first-order and the secondorder interaction in the model of Flory et al. 
for the PDMS $\mathrm{CH}_{3}\left[\mathrm{Si}\left(\mathrm{CH}_{3}\right)_{2}-\mathrm{O}\right]_{x} \mathrm{Si}\left(\mathrm{CH}_{3}\right)_{3}$ $\operatorname{are}^{15}$

$$
\begin{aligned}
& u_{\mathrm{a}}=\left[\begin{array}{ccc}
1 & \sigma & \sigma \\
1 & \sigma & \sigma \omega \\
1 & \sigma \omega & \sigma
\end{array}\right] \\
& u_{\mathrm{f}}=\left[\begin{array}{ccc}
1 & \sigma & \sigma \\
1 & \sigma & 0 \\
1 & 0 & \sigma
\end{array}\right]
\end{aligned}
$$

where the statistical weight $\sigma$ and $\omega$ are

$$
\begin{aligned}
& \sigma=\exp \left(-E_{\sigma} / R T\right) \\
& \omega=\exp \left(-E_{\omega} / R T\right)
\end{aligned}
$$

where taken are 0.244 and 0.175 at $303 \mathrm{~K}$ corresponding to the conformational energy $E_{\sigma}=850 \mathrm{cal} \mathrm{mol}^{-1}$ and $E_{\omega}=1050 \mathrm{cal} \mathrm{mol}^{-1}$, respectively. ${ }^{16}$ The three conformations for the rotational angles $\varphi_{\mathrm{a}}$ and $\varphi_{\mathrm{f}}$ are 0 for the trans form and $\pm 120^{\circ}$ for the gauche forms. Simultaneously, substitution the geometric parameters listed in Table $\mathrm{I}^{17}$ and the masses of $\mathrm{Si}, \mathrm{O}$, and $\mathrm{CH}_{3}$ into eq 36 gives the unperturbed characteristic ratio of meansquare radius of gyration $\left\langle S^{2}\right\rangle / 2 x l^{2} v s$. the degree of polymerization $x$ for the PDMS shown in Figure 2, where $l$ is the length of bond $\mathrm{Si}-\mathrm{O}$. The ratio of $\left\langle S^{2}\right\rangle$ is nearly constant for large $x$. The dependence of mean-square radius of gyration on the molecular weight

$$
\left\langle S^{2}\right\rangle / M=7.82 \times 10^{-2} \AA^{2}(\mathrm{~g} \cdot \mathrm{mol})^{-1}
$$

is in agreement with the experimental data. ${ }^{13}$

$$
\left\langle S^{2}\right\rangle / M=(7.7 \pm 0.3) \times 10^{-2} \AA^{2}(\mathrm{~g} \cdot \mathrm{mol})^{-1}
$$

Table I. The geometric parameter for poly(dimethylsiloxane)

\begin{tabular}{cccc}
\hline Bond and distance & Length $/ \AA$ & Bond angle & Angle $/^{\circ}$ \\
\hline $\mathrm{Si}-\mathrm{O}$ & 1.63 & $\mathrm{Si}-\mathrm{O}-\mathrm{Si}$ & 145.0 \\
$\mathrm{Si}-\mathrm{C}$ & 1.90 & $\mathrm{O}-\mathrm{Si}-\mathrm{O}$ & 110.0 \\
$\mathrm{CH}_{3} \cdots \mathrm{CH}_{3}\left(r_{\mathrm{bd}}\right)$ & 3.10 & $\mathrm{O}-\mathrm{Si}-\mathrm{C}$ & 109.5 \\
& & & \\
\hline
\end{tabular}

The numerical result in eq 40 is dominantly contributed by the first term in eq 36 . The second term can be regarded as the revision considering the effect of side groups. The ratio of $\left\langle S^{2}\right\rangle$ for the PDMS considering the pair $-\left(\mathrm{CH}_{3}\right)_{2}$ is about greater $5 \%$ than that without considering them for $x=100$, and the proportion decrease when $x$ increase. The reason of the side group effect on chain dimension can

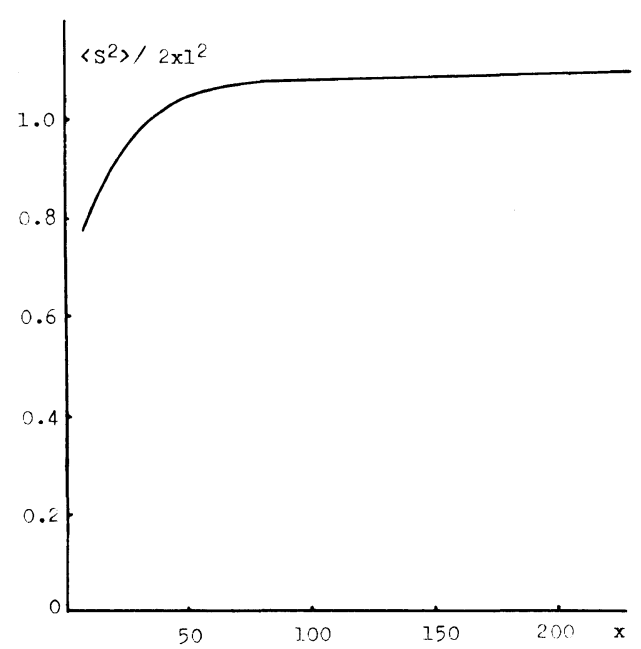

Figure 2. The characteristic ratio of $\left\langle S^{2}\right\rangle / 2 x l^{2} v s$. the degree of polymerization $x$ at $303 \mathrm{~K}$ for poly(dimethylsiloxane) considering the effect of side groups.

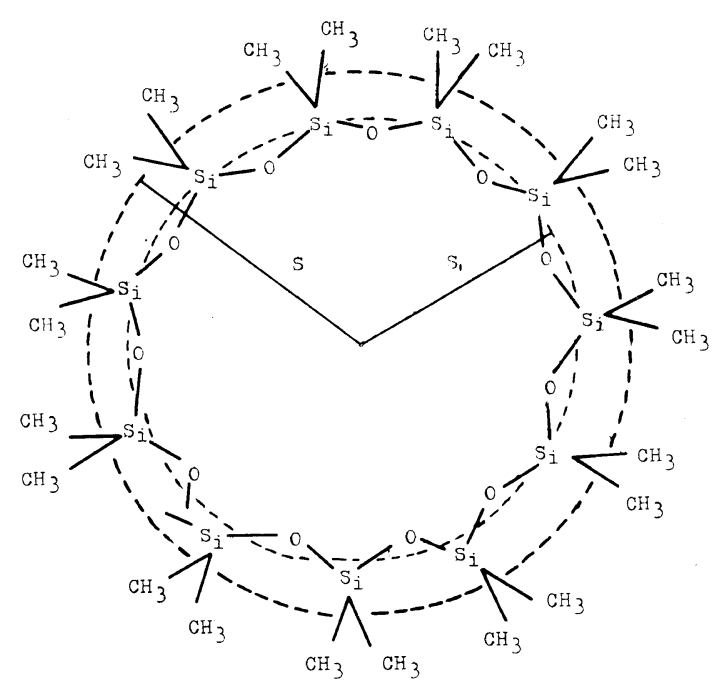

Figure 3. An extended chain of the PDMS molecule in all-trans conformation. 
be traced to the conformational energy and the distribution of the center of mass of monomers for that of chain. As an example of short polymer chain shown in Figure 3, the PDMS chain in the preferred conformation of all-trans closes upon itself after several skeletal bonds. ${ }^{18}$ $S$ and $S_{1}$ represent the distance from the center of mass of chain to the center of masses of each monomer. It seems that the radius $S$ considering $-\left(\mathrm{CH}_{3}\right)_{2}$ is different from the radius $S_{1}$ without considering that. The center of mass of each monomer, besides, is related to the skeletal atoms $\mathrm{Si}$ and $\mathrm{O}$ in other configurations of the PDMS chain. Even though the mass of $-\left(\mathrm{CH}_{3}\right)_{2}$ is $30 / 44.1$ of that of the skeletal atoms $\mathrm{Si}$ and $\mathrm{O}$, and the length of bond $\mathrm{Si}-\mathrm{C}$ is $19.0 / 16.3$ of that of $\mathrm{Si}-\mathrm{O}$, the effect of $-\left(\mathrm{CH}_{3}\right)_{2}$ on $\left\langle S^{2}\right\rangle$ is small because the bond angle $\mathrm{C}-\mathrm{Si}-\mathrm{C}$ is large. If the spatial dimension (considering the shape) and the mass of side groups are larger such as the PDNS, the effect of them on the mean-square radius of gyration may be important. Then, this work gives another method treating the mean-square radius of gyration by the RIS theory for the chain molecules with the pair side groups, especially for the short polymer chain and the polymer chains carrying large side groups.

\section{REFERENCES}

1. Z. Zhiping, X. Jianmin, S. Xubing, and Y. Deyue, Eur. Polym. J., 28, 1339 (1992).

2. Z. Zhiping and Y. Deyue, J. Chem. Phys., 96, 4792 (1992).

3. G. D. Wignall, D. G. H. Ballard, and J. Schelten, Eur. Polym. J., 10, 961 (1974).

4. K. Venkataswamy, A. M. Jamieson, and R. G. Petschek, Macromolecules, 19, 124 (1986).

5. Y. Tamai, T. Konishi, Y. Einaga, M. Fujii, and H. Yamakawa, Macromolecules, 23, 4067 (1990).

6. A. Zirkel, V. Urdor, and D. Richter, Macromolecules, 25, 6148 (1992).

7. P. J. Flory, "Statistical Mechanics of Chain Molecules," Interscience, New York, 1969.

8. P. J. Flory, Macromolecules, 7, 381 (1974).

9. P. J. Flory and D. Y. Yoon, J. Chem. Phys., 61, 5358 (1974).

10. Z. Zhiping, Y. Deyue, and T. Aoqing, Acta Chemica Sinica (Chinese), 50, 313 (1992).

11. Z. Linxi and G. Kuiqi, Eur. Polym. J., 29, 1339 (1993).

12. J. E. Mark, Macromolecules, 11, 627 (1978).

13. J. G. Zilliox, J. E. L. Roovers, and S. Bywater, Macromolecules, 8, 573 (1975).

14. C. L. Lee and F. A. Emerson, J. Polym. Sci., A-2, 5, 829 (1967).

15. P. J. Flory, V. Crescenzi, and J. E. Mark, J. Am. Chem. Soc., 86, 146 (1964).

16. I. Bahar, I. Zuniga, R. Dodge, and W. L. Mattice, Macromolecules, 24, 2993 (1991).

17. I. Bahar, I. Zuniga, R. Dodge, and W. L. Mattice, Macromolecules, 24, 2986 (1991).

18. J. A. Darsey, Macromolecules, 23, 5274 (1990). 